\title{
Estudo das propriedades do concreto convencional com aditivo ou adição de água para correção de consistência
}

\author{
Study of the properties of conventional \\ concrete with additive or addition of \\ water for consistency correction
}

Lucas Damas Maciel ${ }^{1}$, Adenilson Roberto Coelho ${ }^{1}$, Helena Ravache Samy Pereira ${ }^{1}$

\footnotetext{
${ }^{1}$ Centro Universitário Católica de Santa Catarina, Rua Visconde de Taunay, 427, CEP: 89.201-040, Joinville, Santa Catarina, Brasil.

E-mail: lucasdamasmaciel@gmail.com, adenilson_rcoelho@hotmail.com
}

\section{RESUMO}

A fluidez e a plasticidade do concreto devem ser mantidas durante o deslocamento até a obra, impedindo possíveis modificações nas propriedades. Quando há alterações, devem ser corrigidas com água ou aditivos plastificantes. Portanto, esse artigo tem como objetivo avaliar as propriedades de fluidez e resistência característica do concreto. Para isso, produziram-se concretos com fck de $25 \mathrm{MPa}$ e consistência S50. A análise dos resultados deu-se a partir de três experimentos realizados, sendo um sem a correção do Slump Test, o segundo com as verificações de consistência nos tempos de 60, 120 e 150 minutos, logo após o preparo da mistura. Já o terceiro, a verificação consiste no concreto com as mesmas verificações que o segundo, mas, com a adição do aditivo plastificante. Os resultados obtidos apontam que no traço de referência houve minimização da consistência em 64\% ao decorrer dos 150 minutos. A média da resistência à compressão reduziu ao longo do tempo, ao passo que se adicionava água. Logo, a resistência característica caiu em média $7,25 \%$ com relação ao concreto sem a correção do Slump. No entanto, para o concreto com aditivo, a resistência média teve um aumento de $16,52 \%$ durante os 150 minutos. Isso remete ao fato de que o uso de aditivos nos percentuais analisados favorece ao ganho de resistência do concreto. Por outro lado, ao adicionarmos água, o concreto perde resistência. Logo, o uso de água para correção da fluidez não é o ideal, uma vez que altera a resistência final do concreto.

Palavras-chave: Concreto. Aditivo. Consistência. Abatimento.

\begin{abstract}
The fluidity and plasticity of the concrete should be maintained during the displacement to the work, preventing possible changes in the properties. When there are changes, they should be corrected with water or plasticizer additives. Therefore, this is paper aims to evaluate the properties of fluidity and characteristic resistance of concrete. For this, concrete scans were produced with fck equal to $25 \mathrm{MPa}$ and S50 consistency. The analysis of the results occurred from three performed experiments, one without the correction of the Slump Test, the second with consistency checks at times of 60,120 and 150 minutes shortly after the preparation of the mixture. The third, the verification consists of concrete with the same checks as the second, but with the addition of the plasticizer additive. The results obtained indicate that in the reference stroke there was minimization of consistency in $64 \%$ over the 150 minutes. The mean compressive strength decreased over time, while water was added. Therefore, the characteristic resistance fell by $7.25 \%$ compared to concrete without the correction of the Slump. However, for concrete with additive, the average resistance increased by $16.52 \%$ during the 150 minutes. This refers to the fact that the use of additives in the percentages analyzed favors the gain of concrete resistance. On the other hand, when adding water the concrete loses resistance. Therefore, the use of water to correct fluidity is not ideal, since it alters the final resistance of the concrete.
\end{abstract}

Keywords: Concrete. Additive. Consistency. Dispproval. 


\section{INTRODUÇÃO}

Sendo o concreto um dos materiais mais utilizados no mundo, estima-se que o seu consumo esteja na ordem de 11 bilhões de toneladas ao ano [1, 2, 23]. Grande parte do concreto, em muitos países, é proveniente de centrais dosadoras. Portanto, o principal problema na produção de concreto dosado em central é de manter a trabalhabilidade da mistura até o lançamento na obra. O enrijecimento decorrente a cura do concreto agrava-se quanto maior for o tempo da mistura, assim como em condições de tempo com temperaturas elevadas [9, 24, 25, 29].

A minimização de trabalhabilidade no concreto dificulta as operações de mistura, transporte, lançamento, adensamento e acabamento. A compensação da consistência na dosagem do concreto através de adição de água sem o devido controle podem gerar diversas falhas de desempenho do concreto, como por exemplo, ninhos de concreto, diminuição do fator água/cimento $(\mathrm{a} / \mathrm{c})$ e consequentemente a perda de resistência $[1,7,31]$.

Os aditivos estão cada vez mais sendo utilizados nos concretos. Isso é decorrente ao fato de proporcionarem vantagens físicas e econômicas relevantes para a construção civil $[6,23,26]$. Entre os variados tipos de aditivos utilizados, destacam-se no mercado os aditivos redutores de água, classificados como os mais utilizados. Estima-se que 800.000 toneladas destes aditivos são consumidas anualmente em todo mundo [3, 24, 28]. Os aditivos redutores de água reduzem a quantidade de água para uma mesma plasticidade, apresentando melhor trabalhabilidade, menor segregação e melhor condição de vibração e bombeamento [4, 10].

Diversas pesquisas sobre o uso de aditivos redutores de água em materiais cimentícios analisam as vantagens e as desvantagens de sua utilização nos concretos [5, 8, 27]. Os aditivos redutores podem ser utilizados em substituição à água para correção do abatimento ao longo do tempo [11, 30]. Em estudos realizados, utilizou-se o aditivo plastificante para a correção da trabalhabilidade do concreto usinado. Percebeu-se que a correção do abatimento após as 2,5 horas da mistura do concreto reduz em $60 \%$ da resistência mecânica característica [9].

TEIXEIRA [20], ao analisar a correção do abatimento com o uso de água para o concreto com fck de $20 \mathrm{MPa}$, verificou uma perda de resistência à compressão axial na ordem de $44 \%$, com um aumento considerável no fator água/cimento.

Frente isso, o presente artigo tem como objetivo analisar as propriedades de fluidez através da análise de consistência e a resistência mecânica do concreto, adicionando-se a mistura à água ou aditivos plastificantes para a continuidade da consistência requerida.

\section{MATERIAIS E MÉTODOS}

Para as análises, utilizou-se o Laboratório de Materiais de Construção no Centro Universitário Católica de Santa Catarina em Joinville. Na produção dos concretos, empregou-se o cimento CP-II-Z 32 da linha da Votorantim. Como aglomerantes, lidou-se com a areia média proveniente de leito de rio e a brita 1, ambos agregados comercializados em sacos de $20 \mathrm{~kg}$ e da marca Butzke Comercio de Areia LTDA.

A água empregada é proveniente do sistema de abastecimento da rede pública. O aditivo plastificante, Mira 34, é da marca GCP Applied Technologies.

A metodologia de pesquisa pode ser dividida em cinco etapas, conforme a Tabela 1, sendo a caracterização dos materiais constituintes, dosagem do concreto, produção e realização dos ensaios de consistência dos concretos, cura e ensaio de resistência à compressão axial dos corpos de prova.

Tabela 1: Etapas do procedimento experimental

\begin{tabular}{c|c}
\hline ETAPAS & DESCRIÇÃO \\
\hline 1 & Caracterização dos materiais \\
\hline 2 & Dosagem do concreto com fck de 25MPa \\
\hline 3 & Produção dos concretos \\
\hline 4 & Ensaios da consistência \\
\hline 5 & Cura dos concretos e ensaio de resistência à compressão \\
\hline
\end{tabular}

\subsection{Caracterização dos materiais constituintes}

Os agregados foram caracterizados com relação à sua massa específica [13], determinação da massa unitária e do volume agregados [14] e granulometria [12], determinação do material fino passante na peneira $75 \mu \mathrm{m}$, por lavagem dos agregados miúdos [15] e a determinação de impurezas orgânicas nos agregados miúdos [16]. 
Por último, o ensaio de determinação de índice de forma dos agregados graúdos pelo método do paquímetro [17].

\subsection{Dosagem do concreto}

Para a formulação da dosagem de concreto foi utilizado o método ABCP da Associação Brasileira de Cimentos Portland, utilizando para os cálculos as características dos materiais constituintes. Para os ensaios, optou-se pelo concreto convencional da classe C30 e consistência S50 $(90 \pm 10 \mathrm{~mm})$, conforme norma [18].

\subsection{Produção e realização dos ensaios Slump Test}

Produziram-se os concretos em betoneira de 120 litros no decorrer de 150 minutos, período de hidratação e pega da pasta de cimento com os agregados. O equipamento foi desligado somente para o ensaio de abatimento e a moldagem dos Corpos de Prova - CP's.

A produção do concreto foi realizada em três experimentos distintos, conforme Tabela 2. A consistência do concreto foi medida pelo método do ensaio do índice de consistência Slump Test, conforme norma [19].

Tabela 2: Produção do concreto convencional.

\begin{tabular}{c|c}
\hline EXPERIMENTO & DESCRIçÃo \\
\hline 1 & Traço de referência, sem a correção do Slump Test \\
\hline 2 & Traço com adição de água para a correção Slump Test \\
\hline 3 & Traço com adição de aditivo plastificante para a correção do Slump Test \\
\hline
\end{tabular}

\subsection{Cura e ensaio de resistência à compressão dos corpos de prova}

Os procedimentos para a cura do concreto são prescritos pela norma [21], onde os corpos de prova foram desformados após as 24 horas da moldagem. Foram identificados e armazenados em solução saturada de hidróxido de cálcio à temperatura de $(23 \pm 2)^{\circ} \mathrm{C}$, permanecendo no local de cura até a data de ensaio de compressão axial.

Após os 28 dias de cura, os CP's foram submetidos ao ensaio de resistência à compressão na EMIC, modelo PC200CS, NO 11202, conforme a norma [22], obtendo-se a resistência característica fck em MPa.

\section{RESULTADOS}

\subsection{Dosagem do concreto}

O cálculo da dosagem de concreto convencional com fck de 30MPa e abatimento $90 \pm 10 \mathrm{~mm}$, resultou no fator de a/c de 0,45 e desvio padrão de 4,0MPa. O traço final após os cálculos de dosagem são apresentados na Tabela 3. A cada experimento, produziram-se a quantidade de $0,0247 \mathrm{~m}^{3}$ para moldar $12 \mathrm{CP}$ 's, sendo estes para o concreto de referência, o segundo com adição de água e o terceiro com adição de aditivo, totalizando 36 CP's.

Tabela 3: Traço do concreto.

\begin{tabular}{c|c|c|c}
\hline Materiais & Quantidade $\mathbf{( 1 m}^{\mathbf{3}} \mathbf{)}$ & Unidade & $\mathbf{1 2} \mathbf{C P ' s}$ \\
\hline Cimento & $455 \mathrm{~kg}$ & 1,000 & $11,470 \mathrm{~kg}$ \\
\hline Areia & $849 \mathrm{~kg}$ & 1,865 & $20,970 \mathrm{~kg}$ \\
\hline Brita 1 & $925 \mathrm{~kg}$ & 2,032 & $22,850 \mathrm{~kg}$ \\
\hline Água & 205 litros & 0,405 & 05,560 litros \\
\hline
\end{tabular}

As quantidades de água e aditivos utilizados para correção do abatimento são apresentadas pela Tabela 4, para correção da consistência do concreto para os Experimentos 2 e 3 respectivamente. 
Tabela 4: Quantidade de água e aditivo plastificante utilizada no concreto.

\begin{tabular}{c|c|c|c}
\hline \multirow{2}{*}{$\begin{array}{c}\text { Tempo } \\
\text { (minutos) }\end{array}$} & \multicolumn{3}{|c}{ Adição } \\
\cline { 2 - 4 } & Água (ml) & Aditivo (ml) & Aditivo (\%) \\
\hline 00 & - & - & - \\
\hline 60 & 250 & 05,00 & 0,005 \\
\hline 120 & 250 & 10,00 & 0,010 \\
\hline 150 & 100 & 05,00 & 0,005 \\
\hline Total & $\mathbf{6 0 0}$ & $\mathbf{2 0 , 0 0}$ & $\mathbf{0 , 0 2 0}$ \\
\hline
\end{tabular}

\section{2 Índice de consistência do concreto}

Os resultados do índice de consistência do Experimento 1 são apresentados na Tabela 5. Após 150 minutos (min.) do contato entre o cimento e a água, o concreto reduziu em aproximadamente $64 \%$ a sua consistência inicial decorrente à hidratação do cimento e evaporação da água.

Tabela 5: Índice de consistência do Experimento 1.

\begin{tabular}{c|c|c|c}
\hline $\begin{array}{c}\text { Tempo } \\
\text { (min.) }\end{array}$ & $\begin{array}{c}\text { Abatimento } \\
\text { (cm) }\end{array}$ & $\begin{array}{c}\text { Temperatura } \\
\text { (ㅇ) })\end{array}$ & $\begin{array}{c}\text { Umidade Relativa do } \\
\text { Ar (\%) }\end{array}$ \\
\hline 00 & 9,0 & 22,9 & 82 \\
\hline 60 & 7,5 & 24,1 & 60 \\
\hline 120 & 5,5 & 25,8 & 75 \\
\hline 150 & 3,5 & 25,4 & 75 \\
\hline
\end{tabular}

Na Tabela 6, são apresentados os resultados do índice de consistência do Experimento 2. A perda de abatimento foi corrigida adicionando-se a água. Nos primeiros 60 minutos houve uma redução do Slump em 39\%, depois de mais 60 minutos a consistência reduziu em 44\%. Após 30 minutos a redução foi de 33\%.

Tabela 6: Índice de consistência do Experimento 2.

\begin{tabular}{c|c|c|c|c}
\hline Tempo (min.) & Abatimento (cm) & $\begin{array}{c}\text { Abatimento } \\
\text { corrigido }(\mathbf{c m})\end{array}$ & $\begin{array}{c}\text { Temperatura } \\
\mathbf{( \mathbf { o }} \mathbf{C})\end{array}$ & $\begin{array}{c}\text { Umidade Relativa } \\
\text { do } \mathbf{A r}(\%)\end{array}$ \\
\hline 00 & 9,0 & - & 22,8 & 95 \\
\hline 60 & 5,5 & 9,0 & 23,4 & 90 \\
\hline 120 & 5,0 & 9,0 & 24,5 & 84 \\
\hline 150 & 6,0 & 8,5 & 24,8 & 82 \\
\hline
\end{tabular}

Os resultados do índice de consistência do Experimento 3 estão discriminados na Tabela 7. Nos primeiros 60 minutos o abatimento reduziu em 37\%. Passados os 60 minutos, a redução foi de $50 \%$, e, em mais 30 minutos o Slump reduziu em $37 \%$.

Tabela 7: Índice de consistência do Experimento 3.

\begin{tabular}{c|c|c|c|c}
\hline Tempo (min.) & $\begin{array}{c}\text { Abatimento } \\
\text { (cm) }\end{array}$ & $\begin{array}{c}\text { Abatimento } \\
\text { corrigido (cm) }\end{array}$ & $\begin{array}{c}\text { Temperatura } \\
\text { (ํ) }\end{array}$ & $\begin{array}{c}\text { Umidade Relativa } \\
\text { do Ar (\%) }\end{array}$ \\
\hline 00 & 8,0 & - & 29,4 & 76 \\
\hline 60 & 5,0 & 8,0 & 27,7 & 78 \\
\hline 120 & 4,0 & 8,0 & 27,7 & 80 \\
\hline 150 & 5,0 & 7,0 & 28,5 & 79 \\
\hline
\end{tabular}

Os valores de abatimento dos três experimentos são apresentados na (Figura 1), onde o Experimento 1 é denominado como referência, o Experimento 2 como Água e o Experimento 3 como Aditivo.

De acordo com as referências utilizadas [5, 8, 31], a possível perda de consistência inicial do concreto é decorrente à hidratação do cimento e a evaporação da água do interior da pasta de cimento com os agregados. 


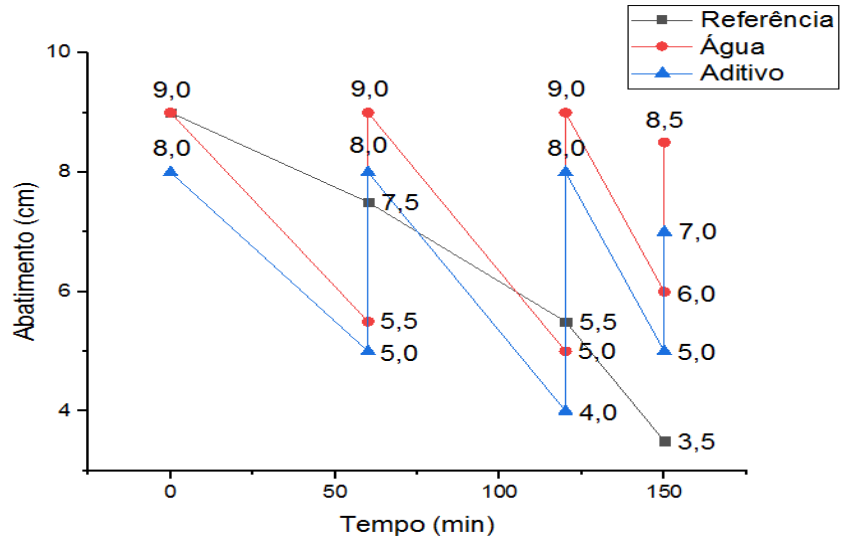

Figura 1: Resultados do Slump Test dos Experimentos.

\subsection{Resistência à compressão do concreto}

Os resultados obtidos para a resistência à compressão aos 28 dias, ao longo do tempo do Experimento 1, são apresentados na (Figura 2). Apesar dos resultados apresentarem um aumento na média da resistência, à medida que se aproximaram aos 150 minutos, é importante observar a variação considerável de desvio padrão.

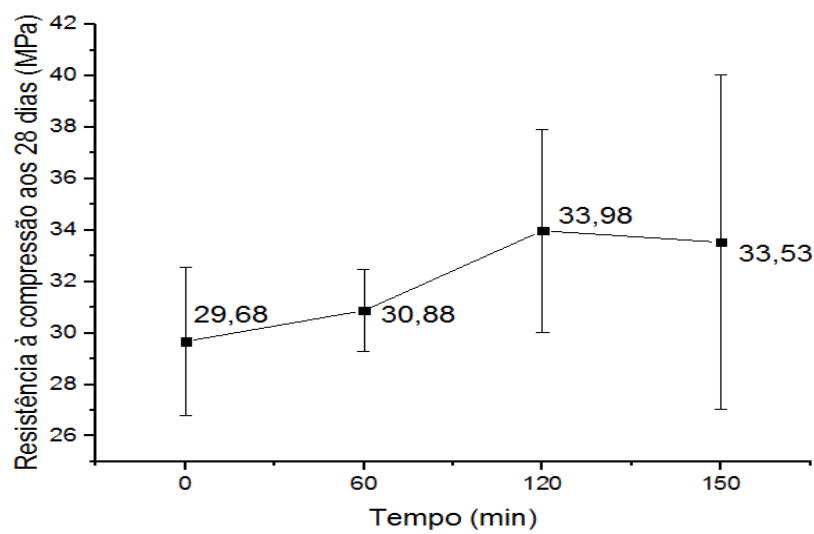

Figura 2: Resultados da resistência à compressão aos 28 dias do Experimento 1 .

A perda de resistência do concreto com a adição de água é apresentada na (Figura 3), totalizando uma redução de aproximadamente 7,25\% em 150 minutos.

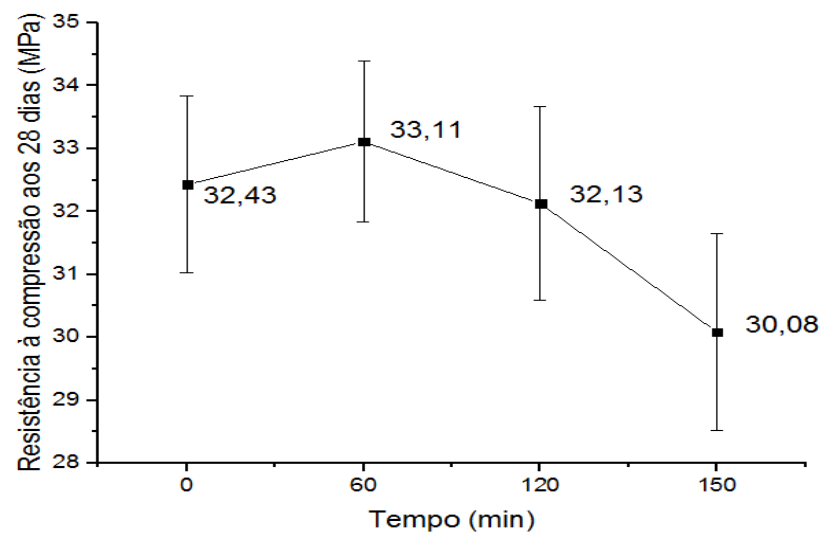

Figura 3: Resultados da resistência à compressão aos 28 dias do Experimento 2.

Os resultados da resistência à compressão aos 28 dias do concreto, com a adição de aditivo, são apresentados na (Figura 4). Pode-se observar um ganho final de 16,52\% de resistência. Salienta-se que a quantidade de água no traço manteve-se a mesma, adicionando-se o aditivo plastificante para a manutenção do abatimento. 


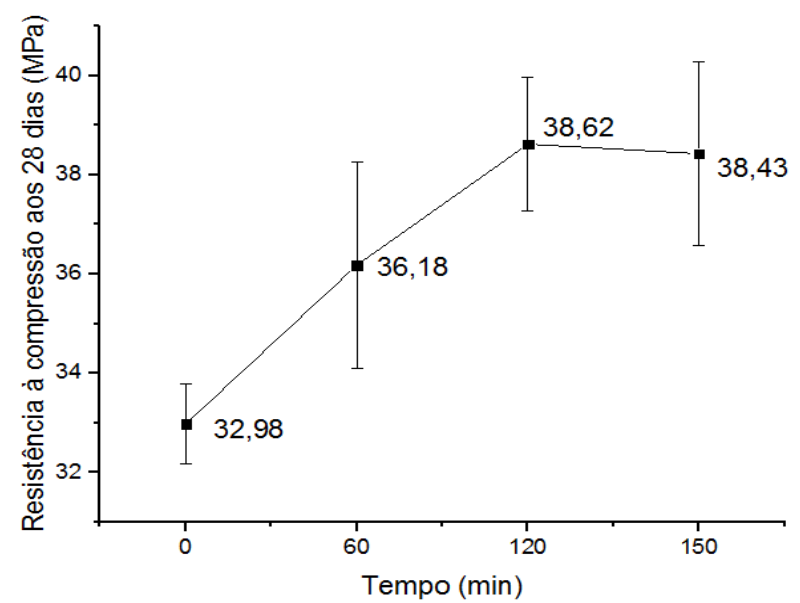

Figura 4: Resultados da resistência à compressão aos 28 dias do Experimento 3.

$\mathrm{Na}$ (Figura 5) são apresentados os resultados de resistência mecânica dos três Experimentos. Para o Experimento 1 pode ser observado um ganho na resistência ao longo do tempo. Contudo, os resultados deveriam ficar bem próximo uns dos outros, pois o traço não sofreu alteração.

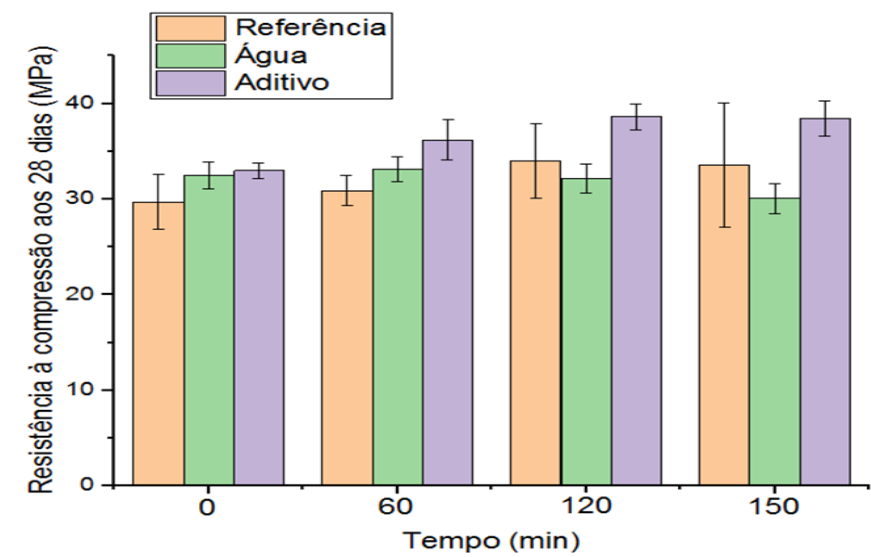

Figura 5: Resultados da resistência à compressão aos 28 dias dos três Experimentos.

\section{DISCUSSÕES}

Ao hidratar a pasta de cimento ocorre aquecimento, logo a água evapora, deixando vazios. Portanto, os poros reduzem o volume do concreto e a consistência inicial. A água por sua vez, interfere diretamente nas ligações da pasta de cimento e no processo de hidratação. Logo, as forças de ligações internas não permanecem na pasta de cimento.

No Experimento 2, com a adição de água, houve uma redução de resistência ao longo do tempo. $\mathrm{O}$ fato ocorreu devido à água estar em excesso para o traço, aumentando o fator a/c. Entretanto, no Experimento 3, com a adição de aditivo plastificante, manifestou-se um ganho na resistência ao longo do tempo.

O aditivo mantem as ligações, não interferindo nas reações químicas e no processo de hidratação. Mantem a água no interior da pasta de cimento, não sendo possível a sua evaporação. Portanto, com o aditivo plastificante a consistência permanece constante, tendendo a aumentar. A não utilização de mais água favorece para que não ocorra perda de resistência do concreto.

Com a adição de água, a resistência característica média baixou para 7,25\% com relação ao concreto sem a correção do Slump. Com acréscimo de aditivo, a resistência média teve um aumento de $16,52 \%$ durante os 150 minutos. Isso remete ao fato de que o uso de aditivos nos percentuais analisados favorece ao ganho de resistência do concreto, não interferindo nas propriedades físicas, comprovando-se as verificações realizadas por autores como [11,30].

Resultados similares no que diz respeito à perda de consistência e a redução de resistência â compressão foi observada por [20]. Ao realizar a correção do abatimento com o uso de água com fck de $20 \mathrm{MPa}$, o concreto apresentou-se com uma perda de $44 \%$ da resistência à compressão axial. Já a consistência teve um aumento 
considerável no fator água/cimento, ou seja, mais água, melhor a consistência e pior resistência.

Em comparação a pesquisas realizadas, como [23, 25, 26], verificou-se também que as misturas com adição de plastificante alcançaram resistências elevadas nos concretos que apresentaram maior trabalhabilidade. Ou seja, o aditivo é ideal para manter-se na consistência desejada sem que prejudique a resistência característica do concreto. Essas evidências também foram verificadas por [27].

\section{CONCLUSÕES}

A verificação da propriedade de consistência do concreto no estado fresco indicou uma redução ao longo do tempo, quando adicionada a água. Já a resistência característica obteve um aumento com a adição do aditivo plastificante. Diante aos dados obtidos, verificou-se que o aditivo plastificante é ideal, pois não altera a resistência do concreto e reduz o consumo de água para o preparo do concreto.

Os resultados do Experimento 1 indicaram uma perda de consistência aos 150 minutos de $64 \%$ no abatimento inicial. Portanto, é justificável o controle das usinas para que o tempo máximo de deslocamento até o local da obra não ultrapasse aos 90 minutos de transporte para veículo dotado de equipamento de agitação.

Para o controle do abatimento, o ideal é o uso de aditivo plastificante, pois o aditivo apresentou uma perda de $37 \%$ em 150 minutos, sem afetar a resistência, já a água, apontou uma perda de 33\%, em 150 minutos. No entanto, o uso da água altera drasticamente a resistência do concreto.

Observou-se que os concretos com a adição suplementar de água apresentaram redução dos valores médios de resistência à compressão, evidenciando a importância do controle para adição de água no concreto.

O uso de aditivos redutores de água ao longo do tempo possibilitou um aumento dos valores médios de resistência à compressão. É importante destacar que a dosagem total de aditivo no concreto não superou a dosagem recomendada pelo fabricante do aditivo, que estabelece o percentual de $1,0 \%$ em relação a massa de cimento.

Como o objetivo do artigo não é verificar as consequências do uso de aditivo em percentual maior do que o estabelecido pelo fabricante, conclui-se que os percentuais utilizados atendem a solicitação do fabricante, assim como, as condições de trabalhabilidade do concreto.

Sendo assim, o estudo da utilização de aditivo plastificante mostrou-se satisfatório e promissor em relação aos resultados, sendo uma ótima alternativa em substituição da adição da água no concreto.

\section{AGRADECIMENTOS}

Ao Centro Universitário Católica de Santa Catarina por disponibilizar os materiais e equipamentos para o preparo do concreto e ensaios. Somos gratos ao técnico do laboratório de Materiais de Construção em Joinville (Robson Rosa) pela ajuda prestada. A empresa GCP Applied Technologies por doar os aditivos utilizados. A esta revista por proporcionar a oportunidade de publicar o artigo.

\section{BIBLIOGRAFIA}

[1] MEHTA, P.K., MONTEIRO, P.J.M. Concreto: Microestrutura, Propriedades e Materiais. 3. Ed. São Paulo: IBRACON, 2008.

[2] NEVILLE, A.M. Propriedades of concrete. Wiley, New York, 1973. Traduzido por Cremonini. R. A. Tecnologia do concreto. 2. Ed. Porto alegre: Bookman editora Ltda., 2016.

[3] BASTOS, A.P.O. "Análise da influência de aditivos superplastificantes no comportamento de pastas de cimento Portland com e sem adição de fíler calcário". Trabalho de Conclusão de Curso - UFRGS, Porto Alegre, RS, 2016.

[4] BAUER, L.A.F. Materiais de construção. 5. Ed. Rio de Janeiro: LTC, 1994.

[5] FOIATO, M.; CERVELIN, S.K. "Avaliação Da Influência Da Combinação Entre Diferentes Tipos De Cimento Com Tipos Distintos De Aditivos Redutores De Água Em Pasta De Cimento". In: XXIV Seminário de Iniciação Científica, Seminário Integrado de Ensino, Pesquisa e Extensão e Mostra Universitária, Joaçaba, SC, 2018.

[6] GRILLO, R.H., et al. "Desempenho comparativo de aditivos redutores de água plastificantes e superplastificantes na produção de concreto". In: $7^{\text {a }}$ Jornada Científica e Tecnológica. IFSUL DE MINAS. Poços de Caldas, MG, 2015.

[7] TOILLIER, H.C. "Estudo do desempenho de aditivos de manutenção de trabalhabilidade no concreto". Trabalho de Conclusão de Curso - UNISC, Santa Cruz do Sul, RS, 2017. 
[8] SCHEEREN, S.C.S., et al. "Influência de aditivos superplastificantes a base de policarboxilato na resistência a compressão e propriedades no estado fresco de um cimento Portland tipo I". Revista de engenharia civil IMED, v.4, n.2, pp. 129-141, Nov. 2017.

[9] FERNANDES, F.M. "Influência da adição de aditivo plastificante e água para manter o abatimento ao longo do tempo na resistência à compressão do concreto". Trabalho de Conclusão de Curso - UNESC, Criciúma, SC, 2011.

[10] BARBOZA, M.R.; BASTOS, P.S. "Traços de concreto para obras de pequeno porte". Concreto e Construção, v.36, pp. 32-36, 2008.

[11] SCHWAAB, S., et al. "Efeito da adição de aditivo plastificante retardador de pega nas propriedades de concretos usinados de cimento Portland usando planejamento fatorial”. Dissertação de M.Sc - UDESC, Joinville, SC, 2015.

[12] ABNT NBR (2003), NBR-248: Determinação da composição granulométrica. Rio de Janeiro, ABNT Associação Brasileira de Normas Técnicas, 2003.

[13] ABNT NBR (2006), NBR-52: Agregado miúdo - Determinação da massa específica e aparente. Rio de Janeiro, ABNT - Associação Brasileira de Normas Técnicas, 2003.

[14] ABNT NBR (2006), NBR-45: Agregados - Determinação da massa unitária e do volume. Rio de Janeiro, ABNT - Associação Brasileira de Normas Técnicas, 2006.

[15] ABNT NBR (2003), NBR-46: Agregado miúdo - Determinação do material fino que passa através da peneira de 75 $\mu \mathrm{m}$. Rio de Janeiro, ABNT - Associação Brasileira de Normas Técnicas, 2003.

[16] ABNT NBR (2001), NBR-49: Agregado miúdo - Determinação de impurezas orgânicas. Rio de Janeiro, ABNT - Associação Brasileira de Normas Técnicas, 2001.

[17] ABNT NBR (2008), NBR-7809: Agregado graúdo - Determinação do índice de forma pelo método do paquímetro - Método de ensaio. Rio de Janeiro, ABNT - Associação Brasileira de Normas Técnicas, 2008.

[18] ABNT NBR (2015), NBR-12655: Concreto de cimento Portland - Preparo, controle, recebimento e aceitação - Procedimentos. Rio de Janeiro, ABNT - Associação Brasileira de Normas Técnicas, 2015.

[19] ABNT NBR (1998), NBR-67: Concreto - Determinação da consistência pelo Abatimento Tronco Cone Métodos de ensaio. Rio de Janeiro, ABNT - Associação Brasileira de Normas Técnicas, 1998.

[20] TEIXEIRA, Renata Baltazar. "Análise da perda de resistência à compressão do concreto com adição de água para correção da perda de abatimento ao longo do tempo". Revista de Iniciação Científica, v. 5, n. 1, pp. 01-15, 2007.

[21] ABNT NBR (2015), NBR-5738: Concreto - Procedimento para moldagem e cura de corpos de prova. Rio de Janeiro, ABNT - Associação Brasileira de Normas Técnicas, 2015.

[22] ABNT NBR (2018), NBR-5738: Concreto - Ensaio de compressão de corpos de prova cilíndricos. Rio de Janeiro, ABNT - Associação Brasileira de Normas Técnicas, 2018.

[23] GUIMARÃES, A.T.C., et al. Materiais de Construção Civil e Princípios de Ciência e Engenharia de Materiais. In ISAIA, Geraldo Cechella. São Paulo: IBRACON. v. 4, n. 2, pp. 984-1022, 2. Ed., Cap. 30, 2010.

[24] NEVILLE, A.M.; BROOKS, J.J. Tecnologia do concreto. 2. Ed. Porto Alegre: Bookman editora Ltda., 2013.

[25] NETO, C.S. Agregados Naturais, Britados e Artificiais para Concreto. In ISAIA, Geraldo Cechella. Concreto: ciência e tecnologia. São Paulo: IBRACON. v. 1, pp. 233-260, Cap. 7, 2011.

[26] HARTMANN, C. Aditivos químicos para concretos e cimentos. In ISAIA, Geraldo Cechella. Concreto: ciência e tecnologia. São Paulo: IBRACON. v. 1, pp. 347-380, Cap. 10, 2011.

[27] BARBOSA, L.S., et al. "Influência do tempo máximo de mistura e transporte especificado pela ABNT NBR 7212:2012 na resistência a compressão de concretos usinados". Revista de Engenharia Civil IMED, v. 4, n. 2, pp. 20-33, Dez. 2017.

[28] ADRIOLO, F.R. Construções de concreto: Manual de práticas para controle e execução. São Paulo: Editora PINI, 1984.

[29] BATtAGIN, A.F. Cimento Portland. In ISAIA, Geraldo Cechella. Concreto: ciência e tecnologia. São Paulo: Ipsis gráfica e editora, pp. 185-232, Cap. 6, 2011.

[30] MELO, K.A.; MARTINS, V.C.; REPETTE, L.W. "Estudo de compatibilidade entre cimento e aditivo redutor de água". Ambiente Construído, Porto Alegre, 2009. 
[31] TORALLES, B.M., et al. "Estudo comparativo de diferentes métodos de dosagem de concretos convencionais". Revista de engenharia e tecnologia. v.. 10, n. 1, pp. 184-198, 2018.

\section{ORCID}

Adenilson Roberto Coelho

https://orcid.org/0000-0003-0499-0382

Lucas Damas Maciel

https://orcid.org/0000-0003-1529-3750

Helena Ravache Samy Pereira

https://orcid.org/0000-0002-2408-1648 